\title{
TerraCheck: Verification of Dedicated Cloud Storage
}

\author{
Zhan Wang ${ }^{1,2}$, Kun Sun ${ }^{2}$, Sushil Jajodia ${ }^{2}$, and Jiwu Jing ${ }^{1}$ \\ 1 State Key Laboratory of Information Security \\ Institute of Information Engineering \\ Chinese Academy of Sciences, Beijing 100093, China \\ \{zwang, jing $\}$ Olois.cn \\ 2 Center for Secure Information Systems \\ George Mason University, Fairfax, VA 22030, USA \\ $\{$ ksun3, jajodia\}@gmu .edu
}

\begin{abstract}
When hardware resources are shared between mutually distrustful tenants in the cloud, it may cause information leakage and bring difficulties to regulatory control. To address these concerns, cloud providers are starting to offer hardware resources dedicated to a single user. Cloud users have to pay more for such dedicated tenancy; however, they may not be able to detect the unexpected misuse of their dedicated storage due to the abstraction layer of the cloud. In this paper, we propose TerraCheck to help cloud users verify if their dedicated storage devices have been misused to store other users' data. TerraCheck detects the malicious occupation of the dedicated device by monitoring the change of the shadow data that are residual bits intentionally left on the disk and are invisible by the file system. When the cloud providers share the dedicated disk with other users, such misuses can be detected since the shadow data will be overwritten and become irretrievable. We describe the theoretical framework of TerraCheck and show experimentally that TerraCheck works well in practice.
\end{abstract}

Keywords: Dedicated Storage, Cloud Security, Verification.

\section{Introduction}

Cloud service significantly reduces costs by multiplexing hardware resources among users 12. The co-resident data belonging to different users may lead to information leakage, which has become a major security concern for cloud users. For instance, a malicious VM is capable of retrieving the encryption keys [22] from a victim VM hosted on the same physical machine. Sensitive information can be compromised through the covert communication channels based on the shared CPU cache 20, memory bus [19, hard disks 15 18] and so on in the cloud.

Cloud providers are starting to offer physically isolated resources in order to lower the entry barrier of cloud adoption for processing sensitive data. For instance, in Amazon cloud [1, Dedicated Instances are a form of EC2 instances

L. Wang and B. Shafiq (Eds.): DBSec 2013, LNCS 7964, pp. 113-127, 2013.

(C) IFIP International Federation for Information Processing 2013 
launched within the Amazon Virtual Private Cloud (Amazon VPC) that runs on hardware dedicated to a single customer. A dedicated instance ensures that the resources, such as CPU, memory, disk storage and network, are isolated physically at the hardware level. Unsurprisingly, the cloud users have to pay more for the dedicated resources than the regular ones.

Although the dedication property is guaranteed by the Service Level Agreement (SLA), a misbehaved cloud provider may fail to meet the isolation requirement due to either accidental configuration error or intentionally reassigning the unallocated resources to other users. As a consequence, the dedicated resource, for example the storage device, will store the data belonging to unexpected users and cause information leakage. Because the cloud users usually can only see a logical view of their resources due to the abstraction layer or the business model of cloud computing [11, they may not be aware of or not be able to detect the violation of the desired dedicated configuration before the security breaches occur.

In this paper, we propose TerraCheck to help cloud users verify if the unallocated disk space has been occupied by undesired users without the cooperation of the cloud provider. We assume that the cloud providers are honest-but-greedy, i.e., trustworthy for managing user's data without violating the data privacy but greedy for allocating the storage resources not being in use by the dedicated user to other tenants. To detect the greedy allocation, TerraCheck places shadow data on the unallocated disk space and verifies the dedication by detecting the change of the shadow information.

Shadow data are portions of the residue left behind when files are deleted from the disk. As such, this data cannot be accessed directly by the file system, but can be recovered using forensic techniques. We group the set of residual bits related to the same original file as "shadow chunk". We record the hash value and physical disk address of each shadow chunk as verification metadata. To verify the integrity of each shadow chunk, we utilize disk forensics techniques to retrieve shadow chunks according to the prior recorded disk addresses. If the shadow chunks cannot be recovered entirely, it indicates that the unallocated disk space has been overwritten and the dedication property is violated.

Our shadow chunk method has two advantages, comparing to simply stuffing the unallocated disk space with void files. First, it makes the cheating behavior of the honest-but-greedy cloud provider very costly. The retrieval of the shadow chunks relies on the physical disk address of each chunk. If the misbehaved cloud providers move the shadow data to some non-dedicated devices and make the shadow data still retrievable, they must map the prior recorded disk address to the addresses of the new device. Instead, accessing files relies on the file system and can be redirected to another device with less effort. Second, shadow data will not affect the normal use of the dedicated device. The attested disk area filled by the shadow chunks remains available for allocation in the view of the file system. However, if the attested disk area is filled by files, it cannot be occupied by the dedicated user immediately. 
We present two schemes for verifying the dedication property of cloud storage. The basic TerraCheck scheme can detect the unexpected occupation of a dedicated storage device with high accuracy by checking the retrievability of every chunk. With sampling, our advanced probabilistic TerraCheck scheme can discover $10 \%$ unexpected occupation of the dedicated storage device with $95 \%$ probability, by randomly challenging 29 chunks. Therefore, a smaller chunk achieves low computational cost, but results in the large storage of metadata. Furthermore, with the help of Bloom filter with $1 \%$ false positive rate, the size of verification metadata can be reduced 5.5 times.

The rest of the paper is organized as follows. In Section 2 , we describe the threat model and general assumptions. Section 3 presents the requirements and operations of the dedication verification. Section 4 describes both the basic and advanced probabilistic TerraCheck schemes. Section 5 implements two schemes and evaluates both the computational and storage costs. Section 6 overviews the related work. Section 7 concludes this paper.

\section{Threat Model and Assumptions}

The dedication property of cloud storage is guaranteed by the terms in SLA. However, a misbehaved cloud provider may fail to meet such dedication requirement due to either accidental configuration errors or intentionally being greedy with the unallocated storage resources: First, configuration error may allocate dedicated storage space to undesired tenants. For instance, in Amazon dedicated instance, the dedication property is enabled by the "Dedicated" attribute configured at the launch time. The "Dedicated" attribute may be silently disabled (e.g., for software update, server migration or testing). Second, a cloud provider may intentionally place the non-frequently accessed data, such as archive data, to the unoccupied disk space where it is supposed to belong to one specific customer.

We consider the misbehaved cloud providers as honest-but-greedy. Honest means that the cloud providers are not motivated to corrupt user's data or violate the data privacy with respect to the business reputation. However, the cloud providers may be greedy for allocating the storage not in used by the dedicated user to other tenants. Although the honest-but-greedy cloud providers are only interested in the large amount of unused disk space belonging to a dedicated user, they cannot control the behavior of the co-resident tenants once the cloud provider accidentally allocates the unoccupied space to another tenant. Co-resident tenants may threaten the security and privacy of the existing user data, such as exploiting covert channels to retrieve encryption key [22] and other sensitive information [15] or violating the access control policy [18].

We also consider the cloud providers are economically rational. Misbehaved cloud providers will not defeat our verification mechanism by paying higher storage overhead. For example, the cloud provider can intecept the write call or monitor the process of placing the shadow data. However, by doing these, the cloud provider has to store the shadow data somewhere else in order to be able 
to response the verification challenge correctly and occupy the dedicated storage at the same time.

We assume the usage of the dedicated storage is well-planned by the user. For example, the user allocates a determined amount of dedicated disk space to each VM. This is a common practice [11] of resource management in the cloud. When the user launches a small number of VMs, only part of the dedicated storage is allocated. The rest of the dedicated storage should be protected from being exploited by other users due to both the security and performance reasons. We refer this part of the disk space as attested area. The disk space being in use by the dedicated user is called occupied area. Additionally, the attested area may scale up and down based on the occupation of the dedicated disk. TerraCheck requires a small amount of trusted disk space for storing verification metadata on the occupied area. We assume the occupied area is trusted, since an honestbut-greedy cloud provider is trustworthy for managing user data.

\section{System Model}

In TerraCheck, both occupied and attested disk spaces are assigned to the user by the cloud provider and under the management of file system. Occupied area is the disk space which has stored user's data; attested area is the empty disk space that is available to be allocated by the user. TerraCheck only focuses on the verification of dedicated storage assigned by the cloud provider rather than the physical disk, since the users of dedicated storage cannot control the disk space that doesn't belong to them. The verification of dedicated storage that solely occupies a physical disk is another research topic, which can be addressed by existing co-residency checking techniques [1817.

We first formalize the model of TerraCheck. Suppose a user $C$ pays and possesses a dedicated disk with the capacity of $s$ in the cloud. The dedicated disk is divided into two areas as shown in Fig. 1, The occupied area with the capacity of $s_{a}$ disk space has been allocated by $C$ for storing the data associated with running VMs or as general purpose storage. We consider occupied area is trusted by $C$ to execute the TerraCheck and store the verification metadata. The attested area with the capacity of $s_{u}$ disk space remains unallocated where $s_{u}=s-s_{a}$. Attested area is the verification target of TerraCheck. When $C$ needs more disk space by increasing the size of occupied area, the size of attested area will shrink accordingly. The goal of TerraCheck is to verify if the attested area has been maliciously taken by other users or the cloud provider.

TerraCheck consists of four major procedures, as shown in Fig. 1. First, it places shadow chunks on the attested area of the target disk. The shadow chunks are deleted files which cannot be accessed from the file system directly by system calls. Instead, shadow chunks can be recovered by disk forensics technique as long as they have not been overwritten. Second, it generates metadata, such as the hash value of the shadow chunks, for monitoring the alternation of shadow chunks. The metadata are stored on the occupied area where it has been allocated for storing the data associated with running VMs or as general purpose 


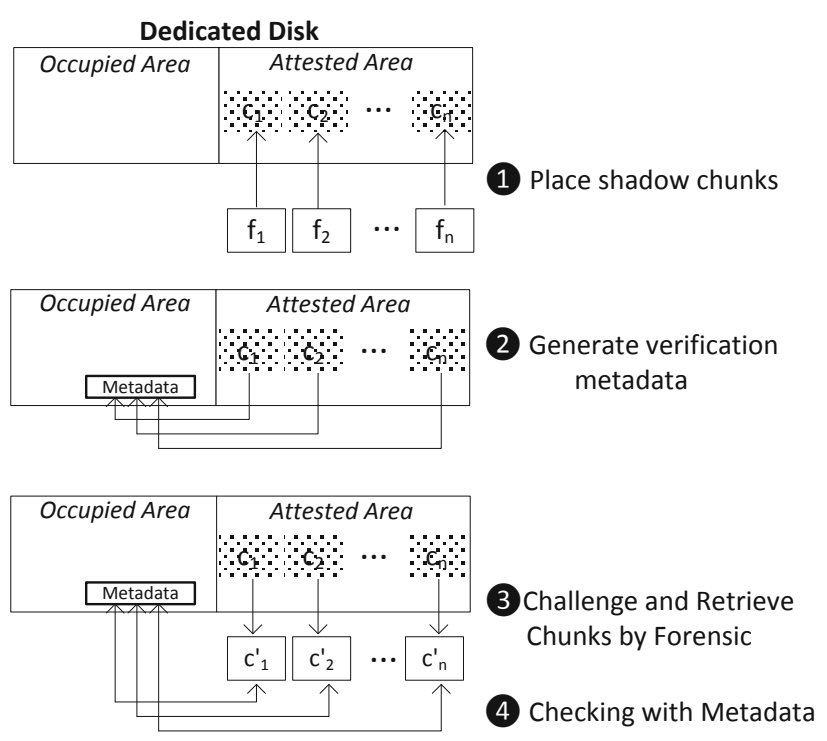

Fig. 1. Overview of TerraCheck

storage. Third, TerraCheck challenges the shadow chunks by using disk forensic techniques to recover them. Lastly, it compares the forensics results with the verification metadata. If any one of the shadow chunks has been altered and cannot be recovered, a violation of dedication property is detected.

\subsection{Verification Requirements}

A solution for verifying the dedicated storage should satisfy the following technical requirements.

- Accuracy. The verification mechanism should ensure the users to trust the result of the verification. When the misbehaved cloud providers break the dedication property by reassigning the dedicated storage to undesired tenants, the user should be able to detect such violation with a high probability.

- Efficiency. The verification procedure should be fast, without obviously interrupting the disk activities against the allocated part of the disk. Moreover, The metadata used for verification should be small; otherwise, it is unacceptable to use the same amount or more disk space to store the original shadow data on the local disk. When the dedicated user occupies or releases more disk space, for example, for running more VMs or shutting down existing VMs, the disk area to be attested varies. Every time the customer needs to scale the disk space up or down, the affected shadow chunks should be as few as possible. 
Table 1. Summary of Operation Parameters

\begin{tabular}{c||l}
\hline Variable & \multicolumn{1}{c}{ Meaning } \\
\hline$C$ & The cloud user who possesses the dedicated device and \\
$n$ & executes dedication verification \\
$l_{k}$ & The number of shadow chunks placed on attested disk area \\
$t_{h}$ & Length of each shadow chunk \\
$t_{f}$ & Header tag of each chunk \\
$K$ & Footer tag of each chunk \\
$s_{u}$ & The set of shadow chunks \\
$i d_{k_{i}}$ & Size of unallocated disk space \\
$F$ & ID of shadow chunk $i$ \\
$i m g_{A A}$ & The set of files for generating shadow chunks \\
$m e t a_{D B}$ & Disk image of attested area \\
$b_{i}$ & File for storing verification metadata \\
$e_{i}$ & Starting disk address of chunk $i$ on attested area \\
$i d_{A R_{x}}$ & Ending disk address of chunk $i$ on attested area \\
$m e t a_{F I L T E R}$ & ID of attested region $x$ \\
\hline
\end{tabular}

\subsection{System Operations}

TerraCheck consists of five basic operations. ChunkGen generates the shadow chunks and places them on the attested area. MetaGen generates the verification metadata and stores them on the occupied area. ChalGen generates the information of challenged chunks. Retrieve executes the forensics of challenged chunks and calculates their hash values. Verify operation compares the result of Retrieve with the verification metadata recorded in MetaGen and makes the decision of the dedication verification. Table 1 summarizes all the variables used in this paper.

- ChunkGen( $\left.n, l_{k}, t_{h}, t_{f}\right) \rightarrow K=\left\{k_{1}, k_{2}, \ldots, k_{n}\right\}:$ TerraCheck fills attested area with a set of chunks $K=\left\{k_{1}, k_{2}, \ldots, k_{n}\right\}$ and $n * l_{k}=s_{u}$. Each chunk $k_{i}$ has a header tag $t_{h}$ and a footer tag $t_{f}$ to represent the start and the end of a chunk, respectively. The total length of the header and the footer $l_{t_{h}}+l_{t_{f}}$ is less than $l_{k}$. This algorithm takes the number of chunks, the length of each chunk, the header $t_{h}$, the footer $t_{f}$ as inputs and generates $n$ temporary files $F=\left\{f_{1}, f_{2}, \ldots, f_{n}\right\}$ first. Every file $f_{i}$ in $F$ starts with $t_{h}$, ends with $t_{f}$ and the rest of it is filled by random bits. Every file $f_{i}$ has the same length as $l_{k}$. All the files in $F$ are stored on attested area and then deleted from the file system. The bits left on attested area associated with each file $f_{i}$ are the set of chunks $K=\left\{k_{1}, k_{2}, \ldots, k_{n}\right\}$. Each chunk contains three parts - the header, the footer, and a random body.

- MetaGen $\left(n, t_{h}, t_{f}, i m g_{A A}, h\right) \rightarrow\left\{\operatorname{meta}_{D B}, \perp\right\}$ : It takes the number of chunks, the header, footer tag information, the disk image of attested area and a hash function as inputs, returns the verification metadata or abortion. $h:\{0,1\}^{*} \rightarrow\{0,1\}^{m}$ denotes a fixed hash function that outputs $m$ bits hash value. The MetaGen algorithm retrieves the chunks from $i m g_{A A}$ by matching the $t_{h}$ and $t_{f}$ and calculates the hash value of each chunk. The results of verification metadata meta $_{D B}$ is stored on occupied area. meta $_{D B}=\left\{\left(i d_{k_{i}}, b_{i}, e_{i}, h\left(k_{i}\right)\right) \mid i \in\{1,2 \ldots n\}, k_{i} \in K\right\}$ lists the ID of a chunk 
and the boundary of each chunk on the disk, such as the start block number $b_{i}$ and the end block number $e_{i}$ of chunk $k_{i}$, and the hash value of each chunk $h\left(k_{i}\right)$. Each chunk can be retrieved from the raw disk based on the start and end block number without the help of the file system. Let $\mid$ meta $_{D B} \mid$ be the number of items in $m e t a_{D B}$. If $\left|m e t a_{D B}\right| \neq n$, it indicates that some chunks either cannot be recovered from the disk image of attested area or a mismatched header or footer involved among the chunks. In this case, MetaGen fails and outputs abortion symbol $\perp$.

- ChalGen $\left(\right.$ met $\left._{D B}, i d_{k_{i}}\right) \rightarrow$ chal: This algorithm generates a challenge chal based on met $_{D B}$ and the ID of the queried chunk. chal $=\left(i d_{k_{i}}, b_{i}, e_{i}, h\left(k_{i}\right)\right) \in$ met $_{D B}$ is the chunk to be examined.

- Retrieve $($ chal, $h) \rightarrow$ result: It takes the challenge and the hash function as inputs and calculates the hash value after retrieving the chunk based on the information specified in chal. It returns the hash value of the chunk in chal.

- Verify (result, chal $) \rightarrow\{$ "success", "failure" $\}$ : The Verify algorithm takes result and chal as inputs and compares the hash value in result with that in chal. If the two hash values match, it outputs "success" and otherwise outputs "failure".

\section{TerraCheck Schemes}

We propose two schemes. The basic TerraCheck can accurately verify the violation of the dedication with a high computation and storage overhead. The advanced TerraCheck can detect the violation of the dedication with a high probability, while reducing the verification overhead dramatically.

\subsection{Basic Scheme}

Our goal is to make sure that the attested area hasn't been allocated to other users. Our basic TerraCheck scheme consists of four phases.

- Initial. In the initial phase, the attested area is filled by all zeros. This operation prevents the existing content on the disk from affecting our placement results.

- Placement. We place the shadow chunks on the attested area by using the ChunckGen and MetaGen algorithms. If MetaGen $\rightarrow \perp$, a failure occurs, TerraCheck should be restarted from the initial phase. Otherwise, MetaGen generates valid verification metadata $\operatorname{met} a_{D B}$.

- Verification is a procedure to patrol on the dedicated storage device and collect the evidence for the undesired occupation by calling Challenge, Retrieve and Verify algorithms until each shadow chunk placed in the attested area has been checked. The Verification phase would be stopped once Verify algorithm returns a "failure" for any chunk. The dedication property is preserved if all the chunks passed the examination. 
- Update is executed when the size of attested area is subject to change. It is difficult to predict the set of affected chunks since the allocation of disk space depends on the disk scheduling. Therefore, both the shadow chunks and their associated verification metadata become useless and subjects to deletion. The initial phase and placement phase should be restarted with the new attested area.

The basic TerraCheck can successfully check the dedication property with high accuracy. If $n-t$ shadow chunks are recoverable, it means that $t$ chunks are altered so that around $t * l_{k}$ out of $s_{u}$ disk space has been allocated or corrupted maliciously. Theoretically, we can $100 \%$ detect the alternation of any number of chunks. However, the basic TerraCheck scheme has two main limitations:

- Computational Cost. The verification phase has to read through the whole attested area and calculate the hash value for every shadow chunk.

- Update Operation. When the size of attested area has to be changed, TerraCheck should be restarted from the initial phase against the new attested area.

\subsection{Advanced Scheme}

To mitigate the limitations of the basic TerraCheck scheme, we propose a probabilistic based TerraCheck scheme. To reduce the computational cost, we randomly sample the chunks during the Verification procedure. In order to provide efficient update operation, we introduce multiple regions within the attested area, we call them attested region. The attested region is the smallest unit for $C$ to scale up the size of the occupied area. For example, $C$ plans to attach a certain size of disk space to a newly launched VM. When the size of the occupied area is shrunk due to the termination of a VM, a new attested region will be created. Each attested region contains multiple shadow chunks. The shadow chunk is the smallest unit for challenge and verification. In addition, we use Bloom filter to reduce the storage for saving the verification metadata.

Attested Region. We introduce attested region for conveniently scaling up and down the size of attested area. The attested area is divided into multiple attested regions. The size of attested region depends on how a user uses the dedicated disk. For example, if it uses the disk as the attached secondary storage for running VMs, and each VM is attached by a fixed amount of disk space, such amount is an optimal size for each attested region. When an attested region should be deleted, the related verification metadata are deleted and excluded from the TerraCheck procedure. The attested region can also serve the purpose of preventing the cloud provider from manipulating the allocation status of the dedicated storage. That is, only the user of dedicated storage can extend the size of occupied area by generating more attested region. 


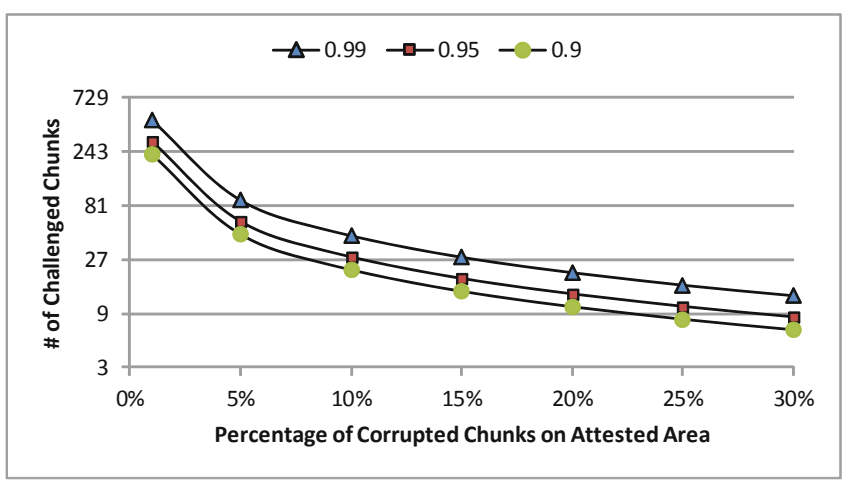

Fig. 2. Probabilistic Framework of Advanced TerraCheck

Probabilistic Verification. The sampling would greatly reduce the computational cost, while still achieving a high detection probability. We now analyze the probabilistic guarantees offered by a scheme that supports chunk sampling.

Suppose the client probes $p$ chunks during the Challenge phase. Clearly, if the cloud provider destroys chunks other than those probed, the cloud provider will not be caught. Assume now that $t$ chunks are tampered and become unrecoverable, so that at least $s_{t}=t * l_{k}$ size of disk space are maliciously allocated. If the total number of chunks is $n$, the probability that at least one of the probed chunks matches at least one of the tampered chunks is $\rho=1-\frac{n-t}{n} \cdot \frac{n-t-1}{n-1}, \ldots, \cdot \frac{n-p+1-t}{n-p+1}$. Since $\frac{n-t-i}{n-i} \geq \frac{n-t-i-1}{n-i-1}$, it follows that $\rho \geq 1-\left(\frac{n-t}{n}\right)^{p}$.

When $t$ is a fraction of the chunks, user $C$ can detect misbehaviors by asking for a constant amount of chunks, independently on the total number of file blocks. As shown in Fig. 2] if $t=1 \%$ of $n$, then TerraCheck asks for 459 chunks, 300 chunks and 230 chunks in order to achieve the probability of at least $99 \%, 95 \%$ and $90 \%$, respectively. When the number of corrupted chunks goes up to $10 \%$ of the total chunks, the violation can be detected with $95 \%$ probability, by only challenging 29 chunks. As the number of corrupted chunks increases, the number of chunks required to be checked is decreased. The sampling is overwhelmingly better than scanning all chunks in the basic TerraCheck scheme. Therefore, we can challenge a fixed number of chunks to achieve certain accuracy. The size of each chunk will determine the computation cost. When the size of each chunk is small, the overhead for retrieving all challenged chunks from dedicated disk is low.

Advanced Operations. For establishing efficient TerraCheck, we need to refine both the MetaGen and ChalGen algorithms.

$\operatorname{MetaGen}\left(n, t_{h}, t_{f}, i m g_{A A}, h\right) \rightarrow\left\{\right.$ meta $\left._{D B}, \perp\right\}$ : The results of verification metadata meta $_{D B}=\left\{\left(i d_{A R_{x}}, i d_{k_{i}}, b_{i}, e_{i}, h\left(k_{i}\right)\right) \mid i \in\{1,2 \ldots n\}, k_{i} \in K\right\}$. It lists the ID of the located attested region, the ID of a chunk and the boundary of each chunk on the disk, such as the start block number $b_{i}$ and the end block 
number $e_{i}$ of chunk $k_{i}$, and the hash value of each chunk $h\left(k_{i}\right)$. Each chunk can be retrieved from the raw disk based on the start and end block number and the ID of the attested region without the help of the file system.

ChalGen $\left(\right.$ meta $\left._{D B}\right) \stackrel{r}{\rightarrow}$ chal. It randomly generates a challenge chal based on meta $_{D B}$. chal $=\left(i d_{A R_{r}}, i d_{k_{r}}, b_{r}, e_{r}, h\left(k_{r}\right)\right) \in$ met $_{D B}$ is the chunk to be examined.

Our advanced TerraCheck scheme consists of the same phases as the basic TerraCheck. Advanced operations will be involved in the related phases, and the update phase should be modified as follows.

- Update. Since the attested area is further divided into attested regions, when a user needs to extend or shrink the disk space for occupied area, only limited number of attested regions are deleted or added so that the TerraCheck against the rest of chunks remains valid. When the occupied area scales up, the metadata related to the erased attested region will be deleted. The rest of metadata are still available for TerraCheck.

Reducing Metadata Storage. In the basic TerraCheck scheme, the size of met $_{D B}$ for storing the verification metadata is linear to the number of shadow chunks. The number of chunks could be very large if the user wants to achieve a lower computational cost, as we discussed in the probabilistic verification. In order to reduce the amount of storage for verification metadata in TerraCheck, we take advantage of Bloom filter to store the metadata for verification.

Bloom filter [4] is a space-efficient data structure for representing a set in order to support membership queries. Bloom filter is suitable to the place where one might like to keep or send a list for verification, but a complete list requires too much space. We use Bloom filter to represent a set $S=\left\{x_{1}, x_{2}, \ldots, x_{n}\right\}$ of $n$ elements as an array of $m$ counters, initially all set to 0 . It uses $k$ independent hash functions $h_{1}, h_{2}, \ldots, h_{k}$ with range $[1, \mathrm{~m}]$. For mathematical convenience, we make the natural assumption that these hash functions map each item in the universe to a random number over the range $\{1, \ldots, m\}$. For each element $x \in S$, the bits $h_{i}(x)$ are set 1 for $1 \leq i \leq k$. A location can be set as 1 multiple times. To check if an item $y$ is a member of $S$, we check whether all $h_{i}(y)$ are 1 . If not, then clearly $y$ is not a member of $S$. If all $h_{i}(y)$ are 1 , we assume that $y$ is in $S$. We know that a Bloom filter may yield a false positive, where it suggests that an element $x$ is in $S$ even though it is not.

The probability of a false positive for an element not in the set, or the false positive rate, can be estimated, given our assumption that hash functions are perfectly random. After all the elements of $S$ are hashed into the Bloom filter, the probability that a specific bit is still 0 is $P R_{z e r o}=1-\frac{1}{m}^{k n} \approx e^{-\frac{k n}{m}}$. The probability of a false positive is $\left(1-P R_{z e r o}\right)^{k}$. A Bloom filter with an optimal value for the number of hash functions can improve storage efficiency.

We modify our TerraCheck model for utilizing Bloom filter to reduce the storage cost of the verification metadata. 
- BF-MetaGen $\left(t_{h}, t_{f}, i m g_{A A}, h\right) \rightarrow\left\{\right.$ meta $\left._{F I L T E R}, \perp\right\}$ The algorithm takes the header, footer tag information, the disk image of attested area and a hash function as inputs, returns the verification metadata or an abortion. meta $_{\text {FILTER }}$ is a Bloom filter which involves the hash value of every shadow chunk.

- BF-Verify(result, meta $\left.{ }_{F I L T E R}\right) \rightarrow\{$ "success", "failure" $\}:$ It takes result and meta FILTER as inputs and checks if the hash value in result is valid and associates with any chunks. If the hash value can be found from meta ${ }_{F I L T E R}$, the algorithm outputs "success" and otherwise "failure".

\section{Implementation and Evaluation}

We implement and evaluate both basic TerraCheck scheme and advanced TerraCheck scheme. All experiments are conducted on a Dell PowerEdge460 server with Intel Core i5 CPU running at 3.10GHz, and with $4096 \mathrm{MB}$ of RAM. The system runs Ubuntu 12.04 (LST) that is configured with Xen Hypervisor. The dedicated storage device is a WestDigital SATA $7200 \mathrm{rpm}$ hard disk with 1TB capacity and $64 \mathrm{MB}$ cache. For evaluation purpose, we used SHA-1 as the hash function $h$. The random values used for challenging the chunks in the advanced TerraCheck are generated using the function proposed by Shoup [7. All data represent the mean of 20 trials.

We implement a large attested area in basic TerraCheck and implement an attested region in advanced TerraCheck as a logical volume. The occupied area may involve multiple logical volumes. LVM (Logical Volume Management) technology is exploited to automate the update operation when the size of the occupied disk space varies. We rely on the retrievability of the shadow chunks on each logical volume to check the dedication property. We utilize Scalpel [14, which is an open source file recovery utility with an emphasis on speed and memory efficiency, to retrieve the shadow chunks based on their header tag and footer tag. To perform file recovery, Scalpel makes two sequential passes over each disk image. The first pass reads the entire disk image and searches for the headers, footers and a database of the locations of these headers. The second pass retrieves the files from the disk image based on the location information of the header and footer. Scalpel is file system-independent and will carve files from FATx, NTFS, ext2 and ext3, or raw partitions.

We evaluate both the computation overhead and storage cost during each phase of TerraCheckand demonstrate the compliance with the requirements of both accuracy and efficiency identified in Section 3.1.

Initial Phase. During the initial phase, the attested area is filled by all zeros. The time for this phase is determined by, and linear to the size of attested area $s_{u}$. It takes about 10 seconds for cleaning $1 \mathrm{~GB}$ of the attested area. Both basic TerraCheck and advanced TerraCheck have the same performance at this phase.

Placement Phase. There are two steps for placing the chunks. The first step is to generate and store the chunks to the attested area. The cost of this operation is 
Table 2. Time for Retrieving Chunks

\begin{tabular}{|c||c|c|c|c|c|c|}
\hline Chunk Size & $512 \mathrm{~KB}$ & $1 \mathrm{MB}$ & $2 \mathrm{MB}$ & $4 \mathrm{MB}$ & $8 \mathrm{MB}$ & $16 \mathrm{MB}$ \\
\hline Retrieve Time & $13 \mathrm{~ms}$ & $15 \mathrm{~ms}$ & $20 \mathrm{~ms}$ & $29 \mathrm{~ms}$ & $48 \mathrm{~ms}$ & $86 \mathrm{~ms}$ \\
\hline
\end{tabular}

determined by the chunk size and the size of the attested area. On our testbed, it takes 12 seconds to store 100MB of shadow chunks. The second step is to generate the metadata. It takes 8.198 seconds for Scalpel to scan 1 GB of the attested area in the first pass and store the location information.

Verification Phase. The basic TerraCheck examines all the chunks based on the verification metadata recorded in meta $_{D B}$. Therefore, the time for generating the challenge can be ignored. The advanced TerraCheck randomly challenges the chunks. The generation of random number takes less than $0.1 \mathrm{~ms}$. The challenged chunks are retrieved from the attested area based on the start and end location recorded as the verification metadata. Therefore, the performance is determined by the disk access time. Tab. 2 shows the disk access time in our experiment.

After retrieving the challenged chunks, TerraCheck compares the hash value of the retrieved chunk with the verification information. In basic TerraCheck, all the chunks residing on the attested area should be checked, which uses the time for calculating the hash value of all the chunks. The advanced TerraCheck scheme randomly challenges the chunks to achieve the detection of undesired disk occupation. We simulate the behaviors that a proportion of attested area is altered. For instance, if a random 1\% of an attested area with 10000 chunks are altered, such a situation could be detected with a $90 \%$ probability by challenging 217 chunks on average, which is close to the theoretical result.

Update Phase. For the basic TerraCheck scheme, the performance of the update is the same as the overhead of executing the initial and placement phases. The performance of the advanced TerraCheck scheme depends on the change of the size of the attested area. When the occupied area is extended, the advanced TerraCheck scheme only needs to update the meta $a_{D B}$ by deleting the items of affected chunks. When the occupied area is shrunk, more attested regions should be created on the attested area. The generation of each attested region takes about $400 \mathrm{~ms}$ regardless the size of the attested region. Therefore, TerraCheck scheme can scale with a low overhead when users update the size of attested area frequently.

Reducing Metadata Storage. apgbmf [2] is originally used to manage Bloom filter for restricting password generation in APG password generation software [16. We use apgbmf version 2.2.3 as a standalone bloom filter management tool.

We consider each hash value of the shadow chunk as an item of password dictionary in the context of apgbmf. We create a Bloom filter for such hash value dictionary. During the verification phase of TerraCheck, if a recovered chunk is unaltered, its hash value will pass the Bloom filter, i.e, the hash value is one of the hash values which associates an original shadow chunk with a high probability. 


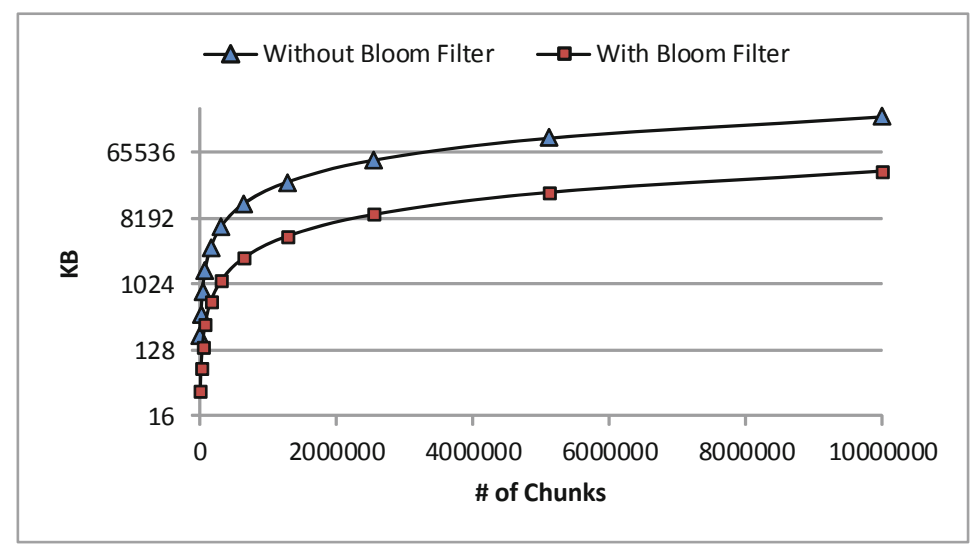

Fig. 3. Comparison of the Storage Cost with/without Bloom Filter ( $\% 1$ Fault Positive Rate Allowed)

When we allow a $1 \%$ fault positive rate, the storage cost with Bloom filter is reduced 5.5 times as shown in Fig. 3. When the number of chunks is more than 10 million, the metadata only requires $36 \mathrm{MB}$ as compared to $200 \mathrm{MB}$ without using Bloom filter.

\section{Related Work}

Cloud service providers [113] are starting to offer physically isolated resources to lower the entry barrier for enterprises to adopt cloud computing and storage. For instance, in Amazon cloud [1], Dedicated Instances are a form of EC2 instances launched within the Amazon Virtual Private Cloud, which runs hardware dedicated to a single customer. Some research has been done to guarantee the exclusive occupation of dedicated resources for security reasons. The side channel based on CPU L2 cache has been used to verify the exclusive use of a physical machine [21. Ristenpart et al. [15] propose to use the existing side channels to verify the co-residency of VMs. 10] allows application designers to build secure applications in the same way as on a dedicated closed platform by using a trusted virtual machine monitor. However, it requires the modification of commercial hypervisor.

Researchers have also investigated techniques to verify various security properties claimed in the SLAs. Dijk et al. 8] prove that the files are stored with encryption at the cloud server side by imposing a resource requirement on the process of translating files from the plain texts to the cipher texts. Proof of Retrievability (PoR) 9] aims to verify if the files are available in the cloud storage at any time. However, PoR cannot verify where the files are located. RAFT [5] can verify that a file is stored with sufficient redundancy by measuring the response time for accessing "well-collected" file blocks. Another work [3] proposes 
a mechanism to verify that the cloud storage provider replicates the data in multiple geo-locations, by measuring the network latency. [18] proposes a method to verify the disk storage isolation of conflict-of-interest files so that Chinese Wall security policy [6] can be successfully enforced in cloud storage environment.

\section{Conclusion}

In this paper, we propose TerraCheck to help cloud users verify the exclusive use of their dedicated cloud storage resources. TerraCheck places shadow chunks on the dedicated disk and detects the change of the shadow information by taking advantage of disk forensics technique. We further improve the computational efficiency by randomly challenging the chunks and reduce the storage by applying Bloom filter.

Acknowledgement. This material is based upon work supported by the National Science Foundation under grant CT-20013A, by US Army Research Office under MURI grant W911NF-09-1-0525 and DURIP grant W911NF-11-1-0340, and by the Office of Naval Research under grant N0014-11-1-0471.

\section{References}

1. Amazon Web Services, http://aws .amazon.com

2. APGBFM, http://linux.die.net/man/1/apgbfm

3. Benson, K., Dowsley, R., Shacham, H.: Do you know where your cloud files are? In: CCSW, pp. 73-82 (2011)

4. Bloom, B.H.: Space/time trade-offs in hash coding with allowable errors. Commun. ACM 13(7), 422-426 (1970)

5. Bowers, K.D., van Dijk, M., Juels, A., Oprea, A., Rivest, R.L.: How to tell if your cloud files are vulnerable to drive crashes. In: ACM Conference on Computer and Communications Security, pp. 501-514 (2011)

6. Brewer, D.F.C., Nash, M.J.: The chinese wall security policy. In: IEEE Symposium on Security and Privacy, pp. 206-214 (1989)

7. Dent, A.W.: The Cramer-Shoup encryption scheme is plaintext aware in the standard model. In: Vaudenay, S. (ed.) EUROCRYPT 2006. LNCS, vol. 4004, pp. 289-307. Springer, Heidelberg (2006)

8. Dijk, M.V., Juels, A., Oprea, A., Rivest, R.L., Stefanov, E., Triandopoulos, N.: Hourglass schemes: How to prove that cloud files are encrypted. In: ACM Conference on Computer and Communications Security (2012)

9. Dodis, Y., Vadhan, S., Wichs, D.: Proofs of retrievability via hardness amplification. In: Reingold, O. (ed.) TCC 2009. LNCS, vol. 5444, pp. 109-127. Springer, Heidelberg (2009)

10. Garfinkel, T., Pfaff, B., Chow, J., Rosenblum, M., Boneh, D.: Terra: a virtual machine-based platform for trusted computing. SIGOPS Oper. Syst. Rev. 37(5), 193-206 (2003)

11. Jhawar, R., Piuri, V.: Fault tolerance management in iaas clouds. In: Proc. of the 1st IEEE-AESS Conference in Europe about Space and Satellite Telecommunications (ESTEL 2012), Rome, Italy (October 2012) 
12. Kurmus, A., Gupta, M., Pletka, R., Cachin, C., Haas, R.: A comparison of secure multi-tenancy architectures for filesystem storage clouds. In: Kon, F., Kermarrec, A.-M. (eds.) Middleware 2011. LNCS, vol. 7049, pp. 471-490. Springer, Heidelberg (2011)

13. Rackspace, http://www.rackspace.com

14. Richard III, G.G., Roussev, V.: Scalpel: A frugal, high performance file carver. In: DFRWS (2005)

15. Ristenpart, T., Tromer, E., Shacham, H., Savage, S.: Hey, you, get off of my cloud: exploring information leakage in third-party compute clouds. In: ACM Conference on Computer and Communications Security, pp. 199-212 (2009)

16. Spafford, E.: Opus: Preventing weak password choices

17. Varadarajan, V., Kooburat, T., Farley, B., Ristenpart, T., Swift, M.M.: Resourcefreeing attacks: Improve your cloud performance (at your neighbor's expense). In: ACM Conference on Computer and Communications Security (2012)

18. Wang, Z., Sun, K., Jajodia, S., Jing, J.: Disk storage isolation and verification in cloud. In: Globecom 2012, Anaheim, CA, USA (2012)

19. Wu, Z., Xu, Z., Wang, H.: Whispers in the hyper-space: High-speed covert channel attacks in the cloud. In: The 21st USENIX Security Symposium (Security 2012) (August 2012)

20. Xu, Y., Bailey, M., Jahanian, F., Joshi, K.R., Hiltunen, M.A., Schlichting, R.D.: An exploration of L2 cache covert channels in virtualized environments. In: CCSW, pp. 29-40 (2011)

21. Zhang, Y., Juels, A., Oprea, A., Reiter, M.K.: Homealone: Co-residency detection in the cloud via side-channel analysis. In: IEEE Symposium on Security and Privacy, pp. 313-328 (2011)

22. Zhang, Y., Juels, A., Reiter, M.K., Ristenpart, T.: Cross-VM side channels and their use to extract private keys. In: Proceedings of the 2012 ACM Conference on Computer and Communications Security, CCS, pp. 305-316 (2012) 